\title{
[COMMODE] a large-scale database of molecular descriptors using compounds from PubChem
}

\author{
Andreas Dander ${ }^{1,2,3}$, Laurin AJ Mueller ${ }^{1}$, Ralf Gallasch ${ }^{1,2,3}$, Stephan Pabinger², Frank Emmert-Streib ${ }^{4}$, \\ Armin Graber ${ }^{1}$ and Matthias Dehmer ${ }^{1 *}$
}

\begin{abstract}
Background: Molecular descriptors have been extensively used in the field of structure-oriented drug design and structural chemistry. They have been applied in QSPR and QSAR models to predict ADME-Tox properties, which specify essential features for drugs. Molecular descriptors capture chemical and structural information, but investigating their interpretation and meaning remains very challenging.
\end{abstract}

Results: This paper introduces a large-scale database of molecular descriptors called COMMODE containing more than 25 million compounds originated from PubChem. About 2500 DRAGON-descriptors have been calculated for all compounds and integrated into this database, which is accessible through a web interface at http://commode.i-med. ac.at.

Keywords: Chemical databases, Molecular descriptors, PubChem, QSAR, QSPR

\section{Background}

Molecular descriptors have been proven essential in drug design, structural and mathematical chemistry, bioinformatics, and related disciplines [1-7]. Descriptors can be understood as functions to map chemical or structural properties of chemicals to positive real numbers. The latter often results in so-called topological or structural descriptors $[7,8]$ by deriving structural features of molecular structures. Following Todeschini et al. [7], we realize that a vast amount of molecular descriptors have been developed; surprisingly, many of their properties have not yet been properly explored. Table 1 lists the existing categories of molecular descriptors calculated using DRAGON [9].

The main contribution of this paper is to provide a largescale, online available database, containing over 25 million chemicals downloaded from the database PubChem $[10,11]$. Our database, called COMMODE (COMpilation of MOlecular DEscriptors), provides a valuable source

\footnotetext{
*Correspondence: matthias.dehmer@umit.at

1 UMIT, Division for Bioinformatics and Translational Research, Eduard

Wallnoefer Zentrum 1, A-6060 Hall in Tyrol, Austria

Full list of author information is available at the end of the article
}

containing descriptor data, which is usually not available at a large scale. As we have already mentioned, the present paper can be regarded as an application explaining the database as well the underlying tool in brief. It would go far beyond the scope of the paper to explain the features and the application of COMMODE in in depth. COMMODE also allows researchers to examine the interpretation or meaning [5] of molecular descriptors.

In the context of topological descriptors $[1,3,5,6]$, this relates to exploring the structural interpretation of such measures. Exemplarily, branching, cyclicity, connectivity and symmetry are plausible interpretations of such indices that have been investigated, see [5,12-15]. Nevertheless, one is far away from possessing a general framework to tackle this problem by using a large number of available descriptors. We believe that COMMODE will prove to be useful to resolve this challenging task successfully. Also, researchers who are active in QSPR and QSAR $[16,17]$ and those developing chemometrics-driven models [18] using descriptor data might utilize this database.

The paper is organized as follows. In the section 'Implementation', we explain the database scheme, and 
Table 1 Groups of molecular descriptors

\begin{tabular}{llr}
\hline Group & Name of the group & Number of descriptors \\
\hline B01 & Constitutional descriptors & 48 \\
B02 & Topological descriptors & 119 \\
B03 & Walk and path counts & 47 \\
B04 & Connectivity indices & 33 \\
B05 & Information indices & 47 \\
B06 & 2D Autocorrelations & 96 \\
B07 & Edge adjacency indices & 107 \\
B08 & Burden eigenvalue descriptors & 64 \\
B09 & Topological charge indices & 21 \\
B10 & Eigenvalue-based indices & 44 \\
B17 & Functional group counts & 154 \\
B18 & Atom-centered fragments & 120 \\
B20 & Molecular properties & 29 \\
B21 & 2D binary fingerprints & 780 \\
B22 & 2D frequency fingerprints & 780 \\
\hline Bhis & 20 & \\
\hline
\end{tabular}

This table lists the different groups for all computed molecular descriptors created by DRAGON. In total 2,489 molecular descriptors were calculated and integrated into COMMODE.

details of the data generation and integration process. The section 'Results and discussion' outlines the usage of our tool with a standard web browser. The article finishes with 'Conclusions'.

\section{Implementation}

\section{Molecular and structural descriptors}

Molecular descriptors encode certain information about chemicals. As a result, special classes of such measures have been developed to emphasize particular aspects of chemicals, e.g., atom types, bond types or structural properties. In particular, molecular descriptors have been proven essential for designing QSPR/QSAR models efficiently $[16,17]$. In this application, we calculated descriptors using of DRAGON [9]. A class of descriptors that has been investigated extensively are topological (or structural) descriptors [5-8]. Clearly, this class itself can be divided in different subcategories such as graph entropy $[8,19]$ representing information-theoretic indices, eigenvalue-based measures [20,21], distance-based measures $[7,22]$ and symmetry-based descriptors [7]. Note that DRAGON has its own categorization of molecular descriptors and, for instance, it does not consider information-theoretic descriptors representing the structural information content of a chemical structure as topological descriptors.

From a practical point of view, molecular descriptors have been used extensively to predict melting and boiling points [23]. Also, other chemical properties such as properties that are important in the drug design process have been used in combination with molecular descriptors. Crucial properties can be ADME-Tox properties (absorption, distribution, metabolism, excretion, and toxicity) influencing different essential aspects of drugs [24]. Examples for molecular descriptors infulencing ADME-Tox properties are the octanol/water partition coefficient ( $\log \mathrm{P})$ [25], the aqueous solubility description (LogS) [26] and the blood-brain barrier permeation $(\operatorname{LogBB})[27]$.

\section{Large-scale database of molecular descriptors}

This section describes the MySQL database scheme and the process of data integration with Java routines, as well as the calculation of the molecular descriptors by using DRAGON. Moreover, the web page, which provides access to the large scale database and is essential for querying the database, and the implemented descriptive analysis, is explained.

COMMODE contains about 25 million chemicals downloaded from the database PubChem and about 2,500 molecular descriptors, which have been calculated for all of those compounds using DRAGON [9]. The database can be accessed through a php-based web application at http://commode.i-med.ac.at [28], where different queries can be started, files with compounds of interest can be uploaded, and results can be exported. Furthermore, additional descriptive values can be calculated and statistically analyzed by means of statistical measures, correlation and uniqueness.

\section{Compounds}

As we used compounds from PubChem, the first step was to download the complete set provided as SDF-files [29] (Structure Data Format). Those files contain 25,051,770 compounds, which vary largely in their size and structure. The mass of the downloaded compounds ranges from 1 to 59750 Dalton with a median value of 383 Dalton, whereby 45 compounds are heavier than 10,000 Dalton. Also the number of heavy atoms (anything other than hydrogen) varies dramatically between 0 and 576 with a median at 27. Other constitutional information about the compounds integrated in COMMODE are number of atoms $(\min =1, \max =982$, $\operatorname{median}=48)$, number of bonds $(\min =0$, $\max =990$, median $=50)$ and the number of rings $(\min =0$, $\max =19$, median $=3$ ) per molecule.

\section{Integration of compounds}

A tailored Java routine has been developed for the correct integration of general information about compounds into a MySQL database [30]. In general, this Java routine reads the data from the unpacked SDF-files and creates one entry per compound with attributes such as the PubChem identifier, exact mass, heavy atom count, SMILES [31] format, and the systematic name for all of the 25 million compounds. Figure 1 shows the database scheme for 


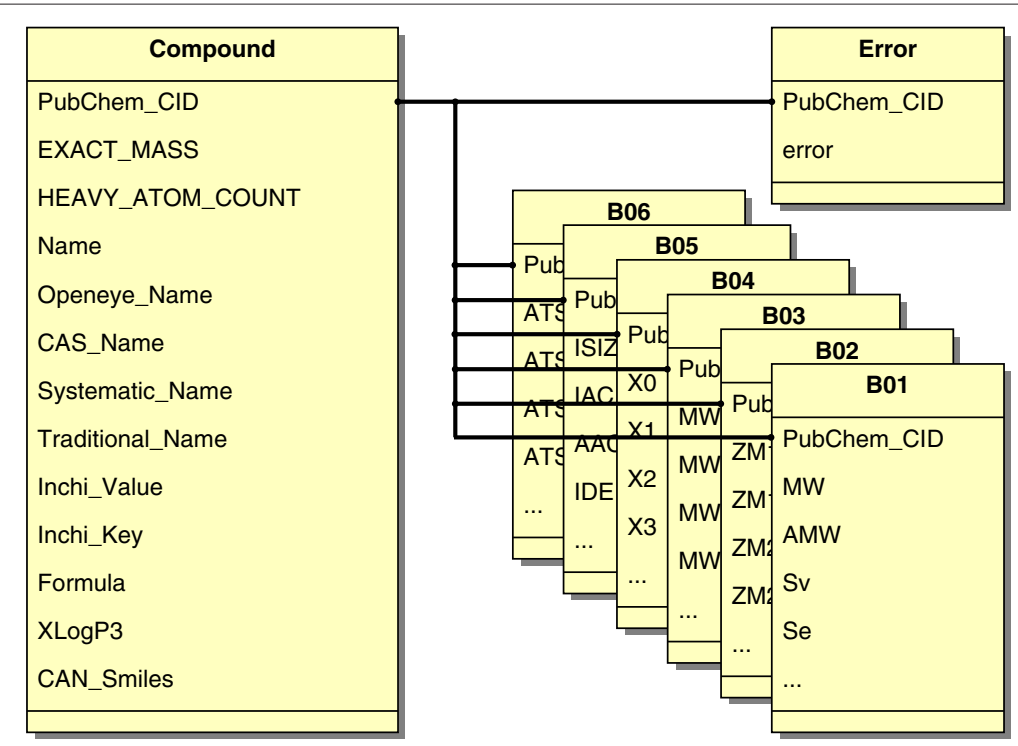

Figure 1 Database scheme for compounds. The main relation in COMMODE is the relation Compound including attributes loaded from PubChem. This relation is linked by using the PubChem identifier with one relation for each of the 15 groups of descriptors (B01-B10, B17, B18, B20-B22) calculated using DRAGON. Each group contains a variety of molecular descriptors (listed in Table 1). For simplification just the first six groups with their corresponding first four descriptors are shown here. Furthermore, the relation Error holds a Boolean value, which is true if a problem occurred using DRAGON for this molecule.

compounds including the central relation Compound containing attributes obtained from PubChem. A second Java routine creates one relation for each single DRAGON group, including molecular descriptors as attributes and the PubChem identifier. All of the mentioned relations are connected to Compound by using PubChem_CID, which is the primary key in those relations.

\section{Computation and integration of molecular descriptors}

After the successful completion of the compound integration, 2,489 molecular descriptors have been calculated by applying DRAGON to all SDF-files. Table 1 lists all 15 calculated groups, their name, and their corresponding number of descriptors. DRAGON produces one file per input file and per group, thereby, numerous files were created holding a huge number of positive real numbers. It appears that DRAGON was not able to calculate the descriptors for 950,688 compounds, due to different errors. These errors can have different causes, like there is just one atom (e.g. hydron $\mathrm{H}^{+}$(id 1038), bromide $\mathrm{Br}^{-}$(id 259)), or the downloaded molecule represents

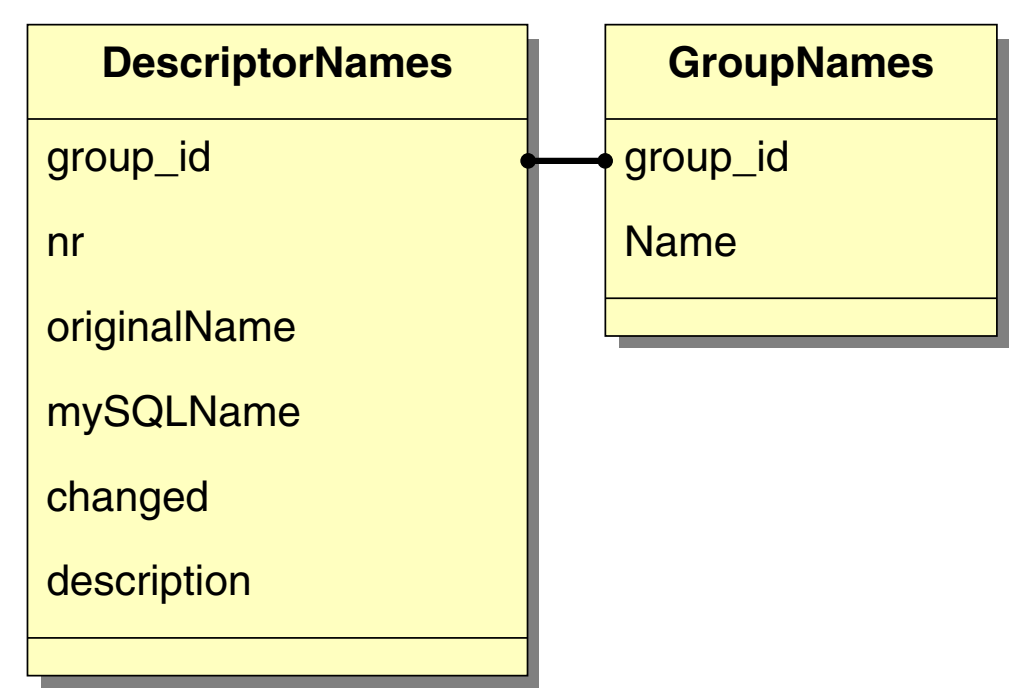

Figure 2 Database scheme for meta data about descriptors and its groups. This part of COMMODE contains general information about descriptors and their corresponding groups integrated in the relations DescriptorNames and GroupNames. 
an unconnected graph and therefore those molecular descriptors can not be computed (e.g. [3-(dimethylcarbamoyloxy)phenyl]-trimethyl-ammonium; methyl sulfate (id 5824)). As DRAGON fails to calculate molecular descriptors for these compounds, the relation Error with the Boolean attribute error was introduced. As 3D information of the compounds is not available using the provided SDF-files, the corresponding 3D descriptors have not been calculated. To handle the large number of result files, an additional Java routine was developed, which integrates those files into the previously generated database relations, where the first attribute for each group contains the identifier from Pubchem.

Meta-data about molecular descriptors and their corresponding groups can also be found in the database scheme. Therefore, the relation DescriptorNames contains attributes describing a molecular descriptor. DescriptorNames is associated with the relation GroupNames, which stores the name of all DRAGON groups. Figure 2 depict those two relations with their corresponding attributes.

\section{Results and discussion Querying COMMODE}

A php-based web application has been developed, which manages the access to the MySQL database. The user can search COMMODE in two different ways. The first way is based on providing different search criteria like the PubChem identifier, exact mass, heavy atom count, different types of name, molecular formula, and ranges for various molecular descriptors. All search criteria are concatenated using a Boolean "AND", which means that

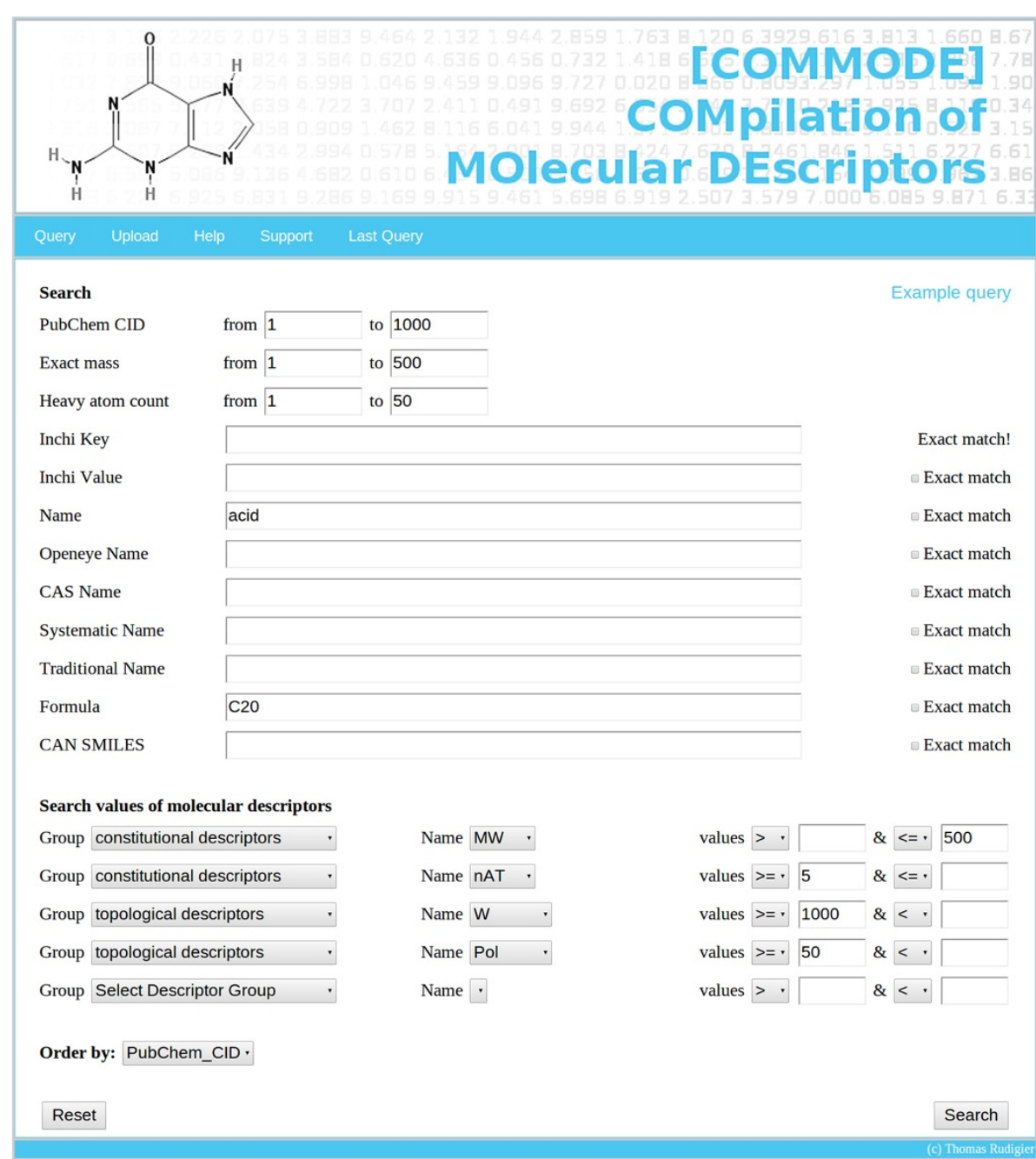

Figure 3 Screen-shot of COMMODE showing the querying page. This screen-shot shows the query page of COMMODE with all different search criteria like PubChem identifier, exact mass, systematic name, smiles, or value ranges for five different molecular descriptors. The results of the given query lists 6 compounds. 
all found molecules must fulfill any given search criteria. Figure 3 shows a screen-shot of the web page including attributes, which are provided for querying the database. The second more flexible way is to provide a CSV-file of interesting compounds using their corresponding PubChem identifier. COMMODE uses this list and is able search for corresponding values of all integrated molecular descriptors. As the database contains around 25 million compounds the resulting tables can be huge, for performance reasons we limit the query result to 1,000 tuples.

\section{Views on the data}

After querying COMMODE various views displaying the results are provided. The first view shows a list with general information about the resulting compounds. Each single molecule can be selected from this list and explored. Therefore, a view was implemented showing different names and values for each molecule as well as a link to the corresponding PubChem page. This view also shows a 2D and a 3D plot from the molecule derived from PubChem. The user can further see the values of all molecular descriptors for the compound of interest or the values

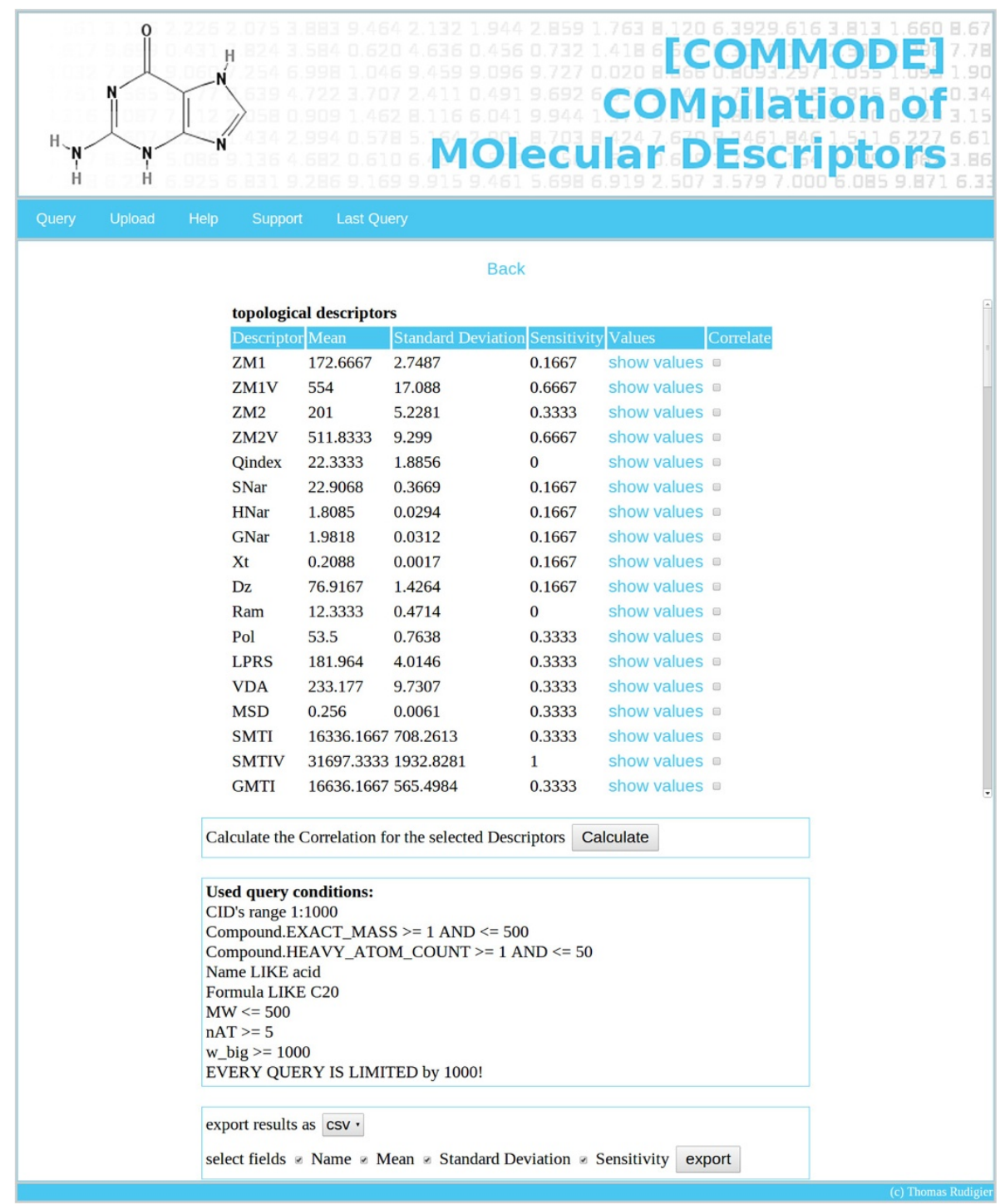

Figure 4 Screen-shot of COMMODE showing the statistics page. This screen-shot shows descriptive statistical values for all topological descriptors of compounds from the query shown in Figure 3. Here each line represents one molecular descriptor with the corresponding mean and standard deviation and the discrimination power (sensitivity) [35,36], all calculated for the queried set of compounds. The 'show values' links can be used to show the values of the given molecular descriptor for all queried compounds, whereas the checkboxes can be used to calculate a correlation matrix between the selected molecules (for the queried compounds). 
from a single molecular descriptor for all compounds of the given query.

\section{Statistical analysis}

When analyzing data of molecular descriptors on a largescale, a statistical analysis is crucial. For example, this relates to estimating the correlations between descriptors to examine whether they capture chemical or structural information of compounds similarly. Note that this problem has been already tackled by Basak et al. [12] and Todeschini et al. [32]; but we would like to emphasize that in their analysis they have only used small subsets of compounds and descriptors. COMMODE offers now the opportunity to investigate this problem on a largescale without the need to having access to a stand-alone application that computes molecular descriptors. This might be particularly interesting for researchers who want to analyze properties of molecular descriptors by using existing compounds.

The same reason described above applies to the analysis of the discrimination power of molecular descriptors. Figure 4 depicts a screen-shot showing molecular descriptors with observed statistical values for the given query. COMMODE is able to estimate Pearson's correlation coefficient. The analysis of the discriminating power of molecular descriptors has not yet been tackled by using large data sets; except for $\mathrm{Hu}$ et al. [33], who investigated the discrimination power of over 4 million structures by calculating EAID numbers. To our knowledge, similar studies have been performed only applying pure structural descriptors by putting the emphasis on rather small sets of compounds [34-36]. Hence, the database system also provides a statistical data analysis part to explore this problem more thoroughly by using a large number of compounds and descriptors.

\section{Data export}

The usage of results within other applications is necessary for scientists, as a lot of downstream analysis can be performed on the integrated data. Therefore, the application supports the following file-formats to export: SDF, SMILES, CSV, MS-Excel and XML format. The connection table of the SDF-file is converted from the stored SMILES code using the Chemistry Development Kit (CDK) [37] and opencsv [38] in a specific Java routine. The exported files can further be used in QSPR and QSAR models.

\section{Conclusions}

This work introduces a large chemical database containing chemical compound data and their corresponding molecular descriptor values. These molecular descriptors can be used in QSPR and QSAR models to predict different chemical parameters using the structure of the compounds, and are utilized in drug design.

The published database, COMMODE, includes more than 25 million compounds and about 2,500 computed descriptors. Clearly, COMMODE extends MOLEdb as this database contains only 1,124 molecular descriptors and 234,773 molecules [39,40]. To use our database in QSPR or QSAR models, compounds of interests can be queried either by using different search attributes or by providing a list of PubChem identifiers. Afterwards, results for molecular descriptors can be exported in different file-formats. These results can further be combined with investigated attributes or properties of the given compounds. New models can be designed using these combinations, which can further be used to predict these attributes and properties for other compounds.

As not all molecular descriptors are necessary for the downstream analysis the introduced application is able to calculate descriptive values for each molecular descriptor representing the discrimination power or the correlation coefficient between chosen descriptors.

An additional research area supported by COMMODE is the field of chemical graph theory [7]. COMMODE can be used to analyze the chemical meaning of molecular descriptors [41]. Therefore, descriptive analysis of all descriptors can be performed for all integrated compounds as well as on a particular subset. Also the degeneracy $[36,42,43]$ of all computed and integrated descriptors can be analyzed on different sets of compounds.

Overall, this novel database provides a flexible access to compounds and their related molecular descriptors, which can be used in different research areas.

\section{Availability and requirements \\ - Project name: COMMODE (COMpilation of MOlecular DEscriptors) \\ - Project home page: $h t t p: / / c o m m o d e . i-m e d . a c . a t$ \\ - Operating system(s): Platform independent \\ - Programming language: Java, php \\ - Other requirements: Web Browser \\ - Any restrictions to use by non-academics: none}

\section{Competing interests}

The authors declare that they have no competing interests.

\section{Authors' contributions}

The conceptual idea for COMMODE by using molecular descriptors goes back to MD. Calculation of all molecular descriptors using DRAGON was performed by MD. Furthermore, MD, AD and FES wrote the manuscript. $A D$ and $L M$ performed the implementation of the database. AD and SP implemented Java routines. RG and AG reviewed the database scheme and performed tests. In summary, the project was initiated and coordinated by MD. All authors contributed to the interpreation of the results and read and approved the final manuscript.

\section{Acknowledgements}

This work was supported by the FFG project Oncotyrol. Matthias Dehmer thanks the Austrian Science Funds for supporting this work (project 
P22029-N13). Also, Matthias Dehmer also gratefully acknowledges funding from the Standortagentur Tirol (formerly Tiroler Zukunftsstiftung).

\section{Author details}

${ }^{1}$ UMIT, Division for Bioinformatics and Translational Research, Eduard Wallnoefer Zentrum 1, A-6060 Hall in Tyrol, Austria. ${ }^{2}$ Biocenter Innsbruck, Division for Bioinformatics, Innsbruck Medical University, Innrain 80-82, A-6020 Innsbruck, Austria. ${ }^{3}$ Oncotyrol, Center for Personalized Medicine, Karl-Kapferer-Straße 5, A-6020 Innsbruck, Austria. ${ }^{4}$ Computational Biology and Machine Learning Laboratory, Center for Cancer Research and Cell Biology, School of Medicine, Dentistry and Biomedical Sciences, Queen's University Belfast, 97 Lisburn Road, Belfast, BT9 7BL, UK.

Received: 30 August 2013 Accepted: 29 October 2013

Published: 13 November 2013

\section{References}

1. Kier LB, Hall LH: Molecular Connectivity in Chemistry and Drug Research. New York, USA: Academic Press; 1976.

2. Mazurie A, Bonchev D, Schwikowski B, Buck GA: Phylogenetic distances are encoded in networks of interacting pathways. Bioinformatics 2008, 24(22):2579-2585.

3. Basak SC, Magnuson VR: Molecular topology and narcosis. Arzeim-Forsch/Drug Design 1983, 33(I):501-503.

4. Varmuza K, Demuth W, Karlovits M, Scsibrany H: Binary substructure descriptors for organic compounds. Croat Chem Acta 2005, 78:141-149.

5. Dehmer M, Varmuza K, Borgert S, Emmert-Streib F: On entropy-based molecular descriptors: statistical analysis of real and synthetic chemical structures. J Chem Inf Model 2009, 49:1655-1663.

6. Bonchev D, Rouvray DH: Complexity in Chemistry, Biology, and Ecology. New York, NY, USA: Mathematical and Computational Chemistry, Springer; 2005.

7. Todeschini R, Consonni V, Mannhold R: Handbook of Molecular Descriptors. Weinheim, Germany: Wiley-VCH; 2002.

8. Bonchev D: Information Theoretic Indices for Characterization of Chemical Structures. Chichester: Research Studies Press; 1983.

9. SRL T: Talete: Dragon. [http://www.talete.mi.it/products/ dragon_description.htm]. Accessed: 11/2012.

10. Bolton EE, Wang Y, Thiessen PA, Bryant SH: PubChem: Integrated platform of small molecules and biological activities. In Annual Reports in Computational Chemistry, Volume 4. Edited by Wang W, Barker N, Simmerling C, Madura JD, Cornell W: American Chemical Society; 2008.

11. NLM: The PubChem project. [http://pubchem.ncbinlm.nih.gov]. Accessed: 11/2012.

12. Basak SC, Balaban AT, Grunwald GD, Gute BD: Topological indices: their nature and mutual relatedness. $J$ Chem Inf Comput Sci 2000, 40:891-898.

13. Dehmer M, Mowshowitz A: A history of graph entropy measures. Inform Sci 2011, 1:57-78.

14. Devillers J, Balaban AT: Topological Indices and Related Descriptors in QSAR and QSPR. Amsterdam, The Netherlands: Gordon and Breach Science Publishers; 1999.

15. Nikolić S, Trinajstić N: Complexity of molecules. $J$ Chem Inf Comput Sci 2000, 40:920-926.

16. Bajorath J: Chemoinformatics: Concepts, Methods, and Tools for Drug Discovery. Totowa, NJ, USA: Methods in Molecular Biology, Humana Press; 2004

17. Guha R: On the interpretation and interpretability of quantitative structure-activity relationship models. J Comput Aided Mol Des 2008, 22(12):857-871.

18. Varmuza K, Filzmoser P: Introduction to Multivariate Statistical Analysis in Chemometrics. Boca Raton, FL, USA: Francis \& Taylor, CRC Press; 2009.

19. Dehmer M: Information processing in complex networks: graph entropy and information functionals. Appl Math Comput 2008, 201:82-94.

20. Dehmer M, Sivakumar L, Varmuza K: Uniquely discriminating molecular structures using novel eigenvalue-based descriptors. MATCH Commun Math Comp Chem 2012, 67:147-172.

21. Estrada E: Characterization of the folding degree of proteins. Bioinformatics 2002, 18:697-704.
22. Skorobogatov VA, Dobrynin AA: Metrical analysis of graphs. Commun Math Comp Chem 1988, 23:105-155.

23. Wiener $\mathrm{H}$ : Structural determination of paraffin boiling points. $J$ Amer Chem Soc 1947, 69:17-20.

24. Talevi A, Goodarzi M, Ortiz EV, Duchowicz PR, Bellera CL, Pesce G, Castro EA, Bruno-Blanch LE: Prediction of drug intestinal absorption by new linear and non-linear QSPR. Euro J Med Chem 2011, 46:218-228.

25. Platts JA, Oldfield SP, Reif MM, Palmucci A, Gabano E, Osella D: The RP-HPLC measurement and QSPR analysis of logPo/w values of several Pt(II) complexes. J Inorgan Biochem 2006, 100(7):1199-1207.

26. Duchowicz PR, Castro EA: QSPR Studies on aqueous solubilities of drug-like compounds. Int J Mol Sci 2009, 10(6):2558-2577.

27. Fan Y, Unwalla R, Denny RA, Di L, Kerns EH, Diller DJ, Humblet C: Insights for predicting blood-brain barrier penetration of CNS targeted molecules using QSPR approaches. J Chem Inform Model 2010, 50(6):1123-1133.

28. Rudigier T: Analytical Molecular Database Search - Eine Web-Applikation zur Analyse molekularer Deskriptoren. Austria: Bachelor Thesis, UMIT; 2011.

29. Dalby A, Nourse JG, Hounshell WD, Gushurst AKI, Grier DL, Leland BA, Laufer J: Description of several chemical structure file formats used by computer programs developed at molecular design limited. J Chem Inform Comput Sci 1992, 32(3):244-255.

30. Oracle: MySQL : The world's most popular open source database. [http://www.mysql.com]. Accessed: 11/2012.

31. Gasteiger J, Engel T(Eds): Chemoinformatics: A Textbook. In Chap. Representation of Chemical Compounds. Weinheim, Germany: WILEY-VCH; 2008:401-437.

32. Todeschini R, Cazar R, Collina E: The chemical meaning of topological indices. Chemomet Intell Laboratory Syst 1992, 15:51-59.

33. $\mathrm{Hu} C Y, X u L:$ On highly discriminating molecular topological index. J Chem Inform Comput Sci 1996, 36:82-90.

34. Diudea MV, llić A, Varmuza K, Dehmer M: Network analysis using a novel highly discriminating topological index. Complexity 2011, 16:32-39.

35. Konstantinova EV, Vidyuk MV: Discriminating tests of information and topological indices. Animals and trees. J Chem Inf Comput Sci 2003, 43(6):1860-1871.

36. Konstantinova E: Information-Theoretic Methods in Chemical Graph Theory. In Towards an Information Theory of Complex Networks. Edited by Dehmer M, Emmert-Streib F, Mehler A. Boston: Birkhäuser; 2011:97-126.

37. Steinbeck C, Han Y, Kuhn S, Horlacher O, Luttmann E, Willighagen E: The chemistry development kit (CDK): an open-source java library for chemo- and Bioinformatics. J Chem inform Comput Sci 2003. 43(2):493-500.

38. Smith G: opencsv. Accessed: 11/2012.

39. Ballabio D, Manganaro A, Consonni V, Mauri A, Todeschini R: Introduction to MOLE DB - on-line molecular descriptors database. MATCH Commun Math Comput Chem 2009, 62:199-207.

40. Ballabio D: MOLE db - Molecular Descriptors Data Base. [http://michem.disat.unimib.it/mole_db]. Accessed: 11/2012

41. Todeschini R, Cazar R, Collina E: The chemical meaning of topological indices. Chemomet and Intell Laboratory Syst 1992, 15:51-59.

42. Dehmer M, Grabner M, Varmuza K: Information indices with high discriminative power for graphs. PLOS ONE 2012, 7:e31214.

43. Hunter PR, Gaston MA: Numerical index of the discriminatory ability of typing systems: an application of Simpson's index of diversity. J Clin Microbiol 1988, 26(11):2465-2466.

doi:10.1186/1751-0473-8-22

Cite this article as: Dander et al: [COMMODE] a large-scale database of molecular descriptors using compounds from PubChem. Source Code for Biology and Medicine 2013 8:22. 